\title{
Optional Surgery? What A Surgeon Must Not Miss!
}

\author{
Badr M I Abdulrauf* \\ Section of Plastic Surgery, Department of Surgery, King Faisal Specialist Hospital and Research Center-Jeddah, Saudi Arabia
}

\author{
*Corresponding author: Badr M I Abdulrauf, MD FRCSC, Program Director Plastic \\ and Reconstructive surgery, Department of Surgery, King Faisal Specialist Hospital \\ and Research Center-Jeddah, Saudi Arabia
}

Received Date: February 11, 2021

Published Date: March 03, 2021

\section{Editorial}

Consultation for an optional surgery and or a semi-aesthetic concern can be quite tricky. Unless certain aspects of the evaluation are fulfilled, patient dissatisfaction related issues might arise at any point following the surgery if considered in the first place. A case scenario is given here as an example in order to discuss what we believe some of the most critical points which should not be missed. A 30 years old man who is coming for possible septo-rhinoplasty. He had a history of nasal fracture reduction following a sports injury in the past and believes he has never been happy with his nose since then.

\section{Goals}

There are always principles which govern us in each anatomical area or topic. These have to be matched with other aspects of the evaluation. Functional and aesthetic complaints besides photos of the patient must be evaluated and documented. It is essential here as a surgeon one can clearly see and appreciate what the patient is seeing or bothering him. Considerations should be given to the aesthetic aspects for male rhinoplasty versus a female as well to the patient's ethnicity and skin type. It is essential to make sure patient is psychologically stable, realistic, decent in his conversations, willing to comply with postoperative instructions and understands the limitations of rhinoplasty. Following this, one may begin formulating the surgical plan and steps to be considered. The surgeon must have developed resonable expertise in nasal surgery for the more severe deformities at first before committing himself or herself in aesthetic septo-rhinoplasty.

\section{Desire}

Many patients may initially consult regarding a blockage of their nasal breathing on either side or both but in essence it is the

cosmetic aspect that they care about equally if not more. Often some patients may assume an aesthetic rhinoplasty as part of the functional surgery as a default. Accordingly, it is important to extract those concerns and find out what is it exactly that he or she dislikes about the nasal shape (hump, deviation, tip, nostrils, etc.), and whether they can prioritize wishes and concerns.

\section{Realism}

One has to discover whether this patient is realistic in his requests regarding the nasal shape or not. As an example, if he is thinking or showing pictures of celebrities' noses that he likes to have then that should raise a red flag! Therefore, it is important for this patient to know, there are limits to what extent nasal shape can be modified and the exact desired outcome cannot be guaranteed.

\section{Psychosocial Aspects}

Seeing a spouse or partner accompanying the patient is somewhat reassuring. In this case of the 30 years old male, it is important to find out about his marital status, or whether he has stable relationship. In fact, it becomes vital to exclude a SIMON (Single Immature Male, Obsessive Narcissistic) category, which is not uncommon. Many of such men would be so much focused on certain body part and correlate it to their success in life and relationships. Apparently, the nose turns out to be the most common part. Failure to succeed in career or relationship would result in blaming the surgery most of the time, even years later down the road. Presence of a relative or a supportive peer witnessing the discussion and informed consent process is always beneficial.

The above case scenario might sound common and routine to many, but it is not exactly straight foreword! There are many pitfalls one may encounter; a novice surgeon might handle the 
case technically as per a textbook but may for instance miss a potentially problematic and or psychologically unstable patient. The patient above for example may believe the way his nasal fracture was handled in the past is the reason for his deformity and suffering rather than the accident which caused the deformity in the first place! The same goes for adult patient with congenital cause of deformity, such as cleft lip related. Therefore, the chances for such patients to be dissatisfied with their rhinoplasty or other surgery is also high. Although Rhinoplasty is often used as a classic example for a medicolegal case, any other facial aesthetic surgery can have similar issues, even for much simpler procedures as with Botox or fillers. Patient counselling and education involves all the above outlined areas, each one is equally important as the rest. It is interesting to realize after several years of practice, proper history taking taught in medical school stands still. More the time invested preoperatively and patient been educated about the procedure; smoother the postoperative course is to be expected, and vice versa. At the end if you get that gut feeling something isn't right about a person or situation, trust it. It must be remembered;" Preoperatively patients observe themselves but post operatively they examine".

When patient chooses to consider a procedure or surgery for an unnecessary condition, consideration to the above 4 points while providing a consultation is invaluable, namely: Goals; Desire; Realism; and Psychosocial aspects (GDRP).

\section{Acknowledgement}

None.

\section{Conflict of Interest}

No conflict of interest. 